\title{
The Application of the Homotopy Analysis Method and the Homotopy Perturbation Method to the Davey-Stewartson Equations and Comparison between Them and Exact Solutions
}

\author{
Hassan A. Zedan, ${ }^{1,2}$ W. Barakati, ${ }^{1}$ and Nada Hamad ${ }^{1}$ \\ ${ }^{1}$ Mathematics Department, Faculty of Science, King Abdul Aziz University, P.O. Box 80203, Jeddah 21589, Saudi Arabia \\ ${ }^{2}$ Mathematics Department, Faculty of Science, Kafr El-Sheikh University, Kafr El-Sheikh, Egypt \\ Correspondence should be addressed to Hassan A. Zedan; hassanzedan2003@yahoo.com
}

Received 20 November 2012; Accepted 1 February 2013

Academic Editor: Hui-Shen Shen

Copyright (C) 2013 Hassan A. Zedan et al. This is an open access article distributed under the Creative Commons Attribution License, which permits unrestricted use, distribution, and reproduction in any medium, provided the original work is properly cited.

\begin{abstract}
We introduce two powerful methods to solve the Davey-Stewartson equations: one is the homotopy perturbation method (HPM) and the other is the homotopy analysis method (HAM). HAM is a strong and easy to use analytic tool for nonlinear problems. Comparison of the HPM results with the HAM results, and compute the absolute errors between the exact solutions of the DS equations with the HPM solutions and HAM solutions are obtained.
\end{abstract}

\section{Introduction}

Nonlinear partial differential equations are useful in describing the various phenomena in disciplines. Apart from a limited number of these problems, most of them do not have a precise analytical solution, so these nonlinear equations should be solved using approximate methods.

The application of the homotopy perturbation method (HPM) $[1,2]$ in nonlinear problems has been devoted by scientists and engineers, because this method continuously deforms a simple problem which is easy to solve into the under study problem which is difficult to solve. The homotopy perturbation method was first proposed by $\mathrm{He}$ [3-6].

The HPM yields a very rapid convergence of the solution series in most cases. The method does not depend on a small parameter in the equation. Using homotopy technique in topology, a homotopy is constructed with an embedding parameter $p \in[0 ; 1]$ which is considered as a "small parameter."

No need to linearization or discretization, large computational work and round-off errors are avoided. It has been used to solve effectively, easily, and accurately a large class of nonlinear problems with approximations. These approximations converge rapidly to accurate solutions [7-10]. The goal of He's homotopy perturbation method was to find a technique to unify linear and nonlinear, ordinary or partial differential equations for solving initial and boundary value problems. The HPM was successfully applied to nonlinear oscillators with discontinuities [4] and bifurcation of nonlinear problem [11]. In [6], a comparison of HPM and homotopy analysis method was made on a simple problem.

In 1992, Liao employed the basic ideas of the homotopy in topology to propose a general analytic method for nonlinear problems, namely, homotopy analysis method (HAM) [1215]. This method has been successfully applied to solve many types of nonlinear problems by others [16-20].

In this paper, we consider the Davey-Stewartson (DS) equations for the function $q=q(x, y, t)$ which are given by (see [21])

$$
\begin{gathered}
i q_{t}+\frac{1}{2} \sigma^{2}\left(q_{x x}+\sigma^{2} q_{y y}\right)+\lambda|q|^{2} q-\phi_{x} q=0, \\
\phi_{x x}-\sigma^{2} \phi_{y y}-2 \lambda\left(|q|^{2}\right)_{x}=0, \quad \lambda= \pm 1, \sigma^{2}= \pm 1 .
\end{gathered}
$$

The case $\sigma=1$ is called the DSI equation, while $\sigma=$ $i$ is the DSII equation. The parameter $\lambda$ characterizes the 
focusing or defocusing case. The Davey-Stewartson I and II are two well-known examples of integrable equations in two space dimensions, which arise as higher dimensional generalizations of the nonlinear shrödinger (NLS) equation, as well as from physical considerations [22]. Indeed, they appear in many applications, for example, in the description of gravity-capillarity surface wave packets in the limit of the shallow water.

Davey and Stewartson first derived their model in the context of water waves, from purely physical considerations. In the context, $q(x, y, t)$ is the amplitude of a surface wave packet, while $\phi(x, y)$ is the velocity potential of the mean flow interacting with the surface wave [22].

In [23], solution of DS equations by (HPM) where the amplitude of a surface wave packet $q$ separated into real and imaginary parts, that is, $q=u(x, y, t)+i v(x, y, t)$. Consequently, the system (1) rewritten in the following form:

$$
\begin{gathered}
u_{t}=-\frac{1}{2} \sigma^{2}\left(v_{x x}+\sigma^{2} v_{y y}\right)-\lambda\left(u^{2}+v^{2}\right) v+\phi_{x} v, \\
v_{t}=\frac{1}{2} \sigma^{2}\left(u_{x x}+\sigma^{2} u_{y y}\right)+\lambda\left(u^{2}+v^{2}\right) u-\phi_{x} u, \\
\phi_{x x}-\sigma^{2} \phi_{y y}-2 \lambda\left(u^{2}+v^{2}\right)_{x}=0, \quad \lambda= \pm 1, \sigma^{2}= \pm 1,
\end{gathered}
$$

with the initial condition

$$
\begin{gathered}
u(x, 0, t)=r \operatorname{sech}[s(x-c t)] \cos \left[k_{1} x+k_{3} t\right], \\
v(x, 0, t)=r \operatorname{sech}[s(x-c t)] \sin \left[k_{1} x+k_{3} t\right], \\
\phi(x, 0, t)=f \tanh [s(x-c t)],
\end{gathered}
$$

where $c=k_{2}+\sigma^{2} k_{1}, r=\sqrt{\left(-2 k_{3}+k_{1}^{2} \sigma^{2}+k_{2}^{2}\right) / \lambda}, s=$ $\sqrt{\left(2 k_{3}+k_{1}^{2} \sigma^{2}+k_{2}^{2}\right) / \sigma^{2}}, f=2 \sigma \sqrt{-\lambda} /\left(1-\sigma^{2}\right), k_{1}, k_{2}$, and $k_{3}$ are arbitrary constants.

In this paper, we apply homotopy analysis method (HAM) for the above system. We rewrite system (1) in the following form

$$
\begin{gathered}
\rho_{t}=\sigma^{2} m\left(\theta_{x} \rho_{x}+\frac{1}{2} \rho \theta_{x x}\right)+m\left(\theta_{y} \rho_{y}+\frac{1}{2} \rho \theta_{y y}\right), \\
\theta_{t}=\frac{-1}{2 m \rho}\left(\sigma^{2} \rho_{x x}+\rho_{y y}\right)+\frac{m}{2}\left(\sigma^{2} \theta_{x}^{2}+\theta_{y}^{2}\right)-\frac{1}{m}\left(\lambda \rho^{2}+\phi_{x}\right), \\
\phi_{x x}-\sigma^{2} \phi_{y y}-4 \lambda \rho \rho_{x}=0,
\end{gathered}
$$

where we take $q=\rho(x, y, t) \cdot e^{-m i \theta(x, y, t)}$ with the initial condition

$$
\begin{gathered}
\rho(x, 0, t)=r \operatorname{sech}[s(x-c t)] \cos \left[k_{1} x+k_{3} t\right], \\
\theta(x, 0, t)=r \operatorname{sech}[s(x-c t)] \sin \left[k_{1} x+k_{3} t\right], \\
\phi(x, 0, t)=f \tanh [s(x-c t)],
\end{gathered}
$$

where $c=k_{2}+\sigma^{2} k_{1}, r=\sqrt{\left(-2 k_{3}+k_{1}^{2} \sigma^{2}+k_{2}^{2}\right) / \lambda}, s=$ $\sqrt{\left(2 k_{3}+k_{1}^{2} \sigma^{2}+k_{2}^{2}\right) / \sigma^{2}}, f=2 \sigma \sqrt{-\lambda} /\left(1-\sigma^{2}\right), k_{1}, k_{2}$, and $\mathrm{k}_{3}$ are arbitrary constants. After that we will apply homotopy perturbation method and homotopy analysis method. When implementing the homotopy perturbation method (HPM) and the homotopy analysis method (HAM), we get the explicit solutions of the DS equations without using any transformation method. Furthermore, we will show that considerably better approximations related to the accuracy level would be obtained. The homotopy perturbation method can be found in $[1-11,23]$. The homotopy analysis method can be found in details in [12-22, 24-26] and only the main steps will be summarized here.

\section{Application of the Homotopy Perturbation Method}

To investigate the traveling wave solution of (4), we first construct a homotopy as follows:

$$
\begin{gathered}
\frac{\partial^{2} V_{1}}{\partial y^{2}}-\frac{\partial^{2} v_{1,0}}{\partial y^{2}}+p \frac{\partial^{2} v_{1,0}}{\partial y^{2}} \\
+p\left[2 m V_{1} \frac{\partial V_{2}}{\partial t}+\sigma^{2}\left(-m^{2} V_{1}\left(\frac{\partial V_{2}}{\partial x}\right)^{2}+\frac{\partial^{2} V_{1}}{\partial x^{2}}\right)\right. \\
\left.-m^{2} V_{1}\left(\frac{\partial V_{2}}{\partial y}\right)^{2}+2 \lambda V_{1}^{3}+2 V_{1} \frac{\partial V_{3}}{\partial x}\right]=0 \\
\frac{\partial^{2} V_{2}}{\partial y^{2}}-\frac{\partial^{2} v_{2,0}}{\partial y^{2}}+p \frac{\partial^{2} v_{2,0}}{\partial y^{2}} \\
+p\left[\frac{-2}{m V_{1}} \frac{\partial V_{1}}{\partial t}+\frac{2 \sigma^{2}}{V_{1}}\left(\frac{\partial V_{2}}{\partial x} \frac{\partial V_{1}}{\partial x}+\frac{1}{2} V_{1} \frac{\partial^{2} V_{2}}{\partial x^{2}}\right)\right. \\
\left.+\frac{2}{V_{1}} \frac{\partial V_{2}}{\partial y} \frac{\partial V_{1}}{\partial y}\right]=0, \\
\frac{\partial^{2} V_{3}}{\partial y^{2}}-\frac{\partial^{2} v_{3,0}}{\partial y^{2}}+p \frac{\partial^{2} v_{3,0}}{\partial y^{2}}+p\left[-\frac{1}{\sigma^{2}} \frac{\partial^{2} V_{3}}{\partial x^{2}}+\frac{4 \lambda}{\sigma^{2}} V_{1} \frac{\partial V_{1}}{\partial x}\right]=0
\end{gathered}
$$

And the initial approximations are as follows:

$$
\begin{aligned}
& v_{1,0}(x, y, t)=\rho_{0}(x, y, t)=\rho(x, 0, t), \\
& v_{2,0}(x, y, t)=\theta_{0}(x, y, t)=\theta(x, 0, t), \\
& v_{3,0}(x, y, t)=\phi_{0}(x, y, t)=\phi(x, 0, t), \\
& V_{1}=v_{1,0}+p v_{1,1}+p^{2} v_{1,2}+p^{3} v_{1,3}+\cdots, \\
& V_{2}=v_{2,0}+p v_{2,1}+p^{2} v_{2,2}+p^{3} v_{2,3}+\cdots, \\
& V_{3}=v_{3,0}+p v_{3,1}+p^{2} v_{3,2}+p^{3} v_{3,3}+\cdots,
\end{aligned}
$$




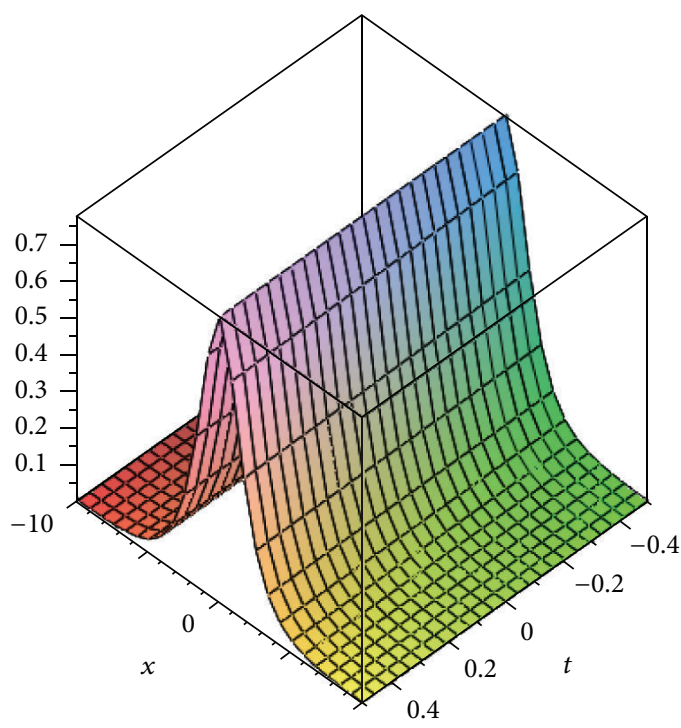

The exact solution of $|q(x, y, t)|$

(a)

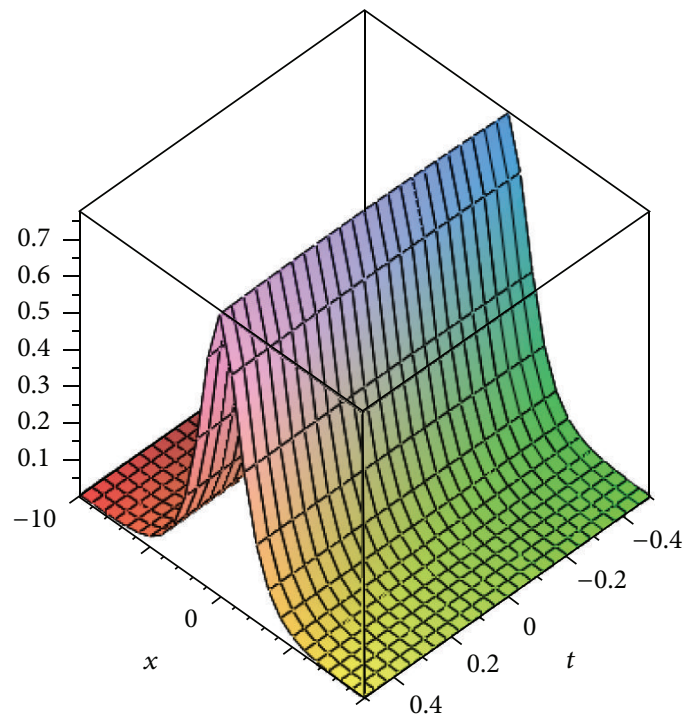

HPM solution for $|q|=\left|\rho e^{-0.1 i \theta}\right|$

(c)

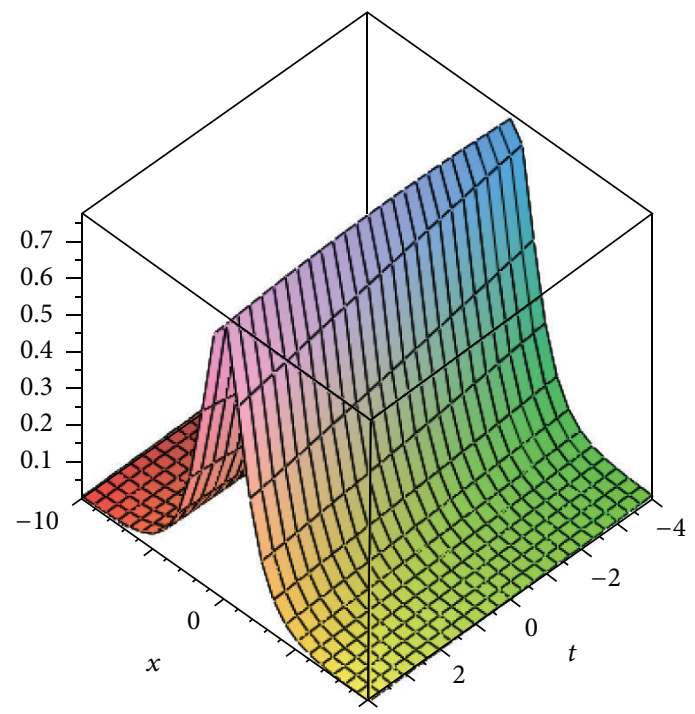

HAM solution for $|q|=|u+i v|$

(b)

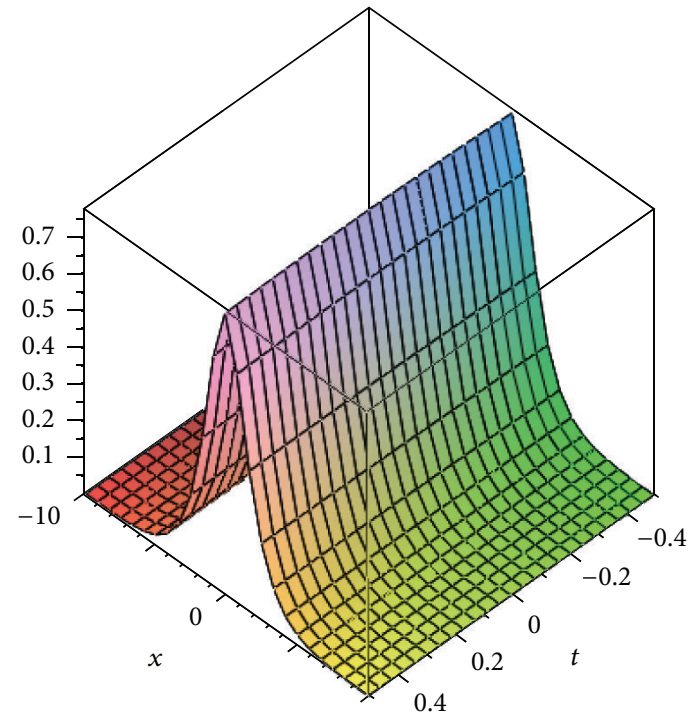

HAM solution for $|q|=\left|\rho e^{-0.1 i \theta}\right|$

(d)

FIgURE 1: Comparison between the exact solution, the HPM solution, and the HAM solution for $q(x, y, t)$.

where $v_{(i, j)}, i=1,2,3, j=0,1,2,3, \ldots$ are functions yet to be determined. Substituting (8) into (6) and arranging the coefficients of $p$ powers, we have

$$
\begin{aligned}
0=( & -2 v_{1,0 t}+m v_{1,0} v_{2,1 y y}+2 \sigma^{2} m v_{2,0 x} v_{1,0 x} \\
+ & \left.\sigma^{2} m v_{2,0 x x} v_{1,0}+m v_{2,0 y y} v_{1,0}+2 m v_{2,0 y} v_{1,0 y}\right) p \\
+ & \left(\sigma^{2} m v_{2,0 x x} v_{1,1}+2 \sigma^{2} m v_{2,1 x} v_{1,0 x}+\sigma^{2} m v_{2,1 x x} v_{1,0}\right. \\
& +m v_{2,1 y y} v_{1,1}-2 v_{1,1 t}+m v_{2,0 y y} v_{1,1} \\
& +2 \sigma^{2} m v_{2,0 x} v_{1,1 x}+m v_{2,2 y y} v_{1,0}
\end{aligned}
$$

$$
\begin{aligned}
& \left.+2 m v_{2,0 y} v_{1,1 y}+2 m v_{2,1 y} v_{1,0 y}\right) p^{2} \\
& +\left(2 m v_{2,2 y} v_{1,0 y}+2 \sigma^{2} m v_{2,0 x} v_{1,2 x}+2 \sigma^{2} m v_{2,1 x} v_{1,1 x}\right. \\
& +m v_{2,1 y y} v_{1,2}-2 v_{1,2 t}+m v_{2,0 y y} v_{1,2} \\
& +\sigma^{2} m v_{2,0 x x} v_{1,2}+\sigma^{2} m v_{2,1 x x} v_{1,1} \\
& +m v_{2,3 y y} v_{1,0}+v_{2,2 y y} m v_{1,1} \\
& +2 m v_{2,0 y} v_{1,2 y}+2 \sigma^{2} m v_{2,2 x} v_{1,0 x} \\
& \left.+2 m v_{2,1 y} v_{1,1 y}+\sigma^{2} m v_{2,2 x x} v_{1,0}\right) p^{3}+\cdots
\end{aligned}
$$




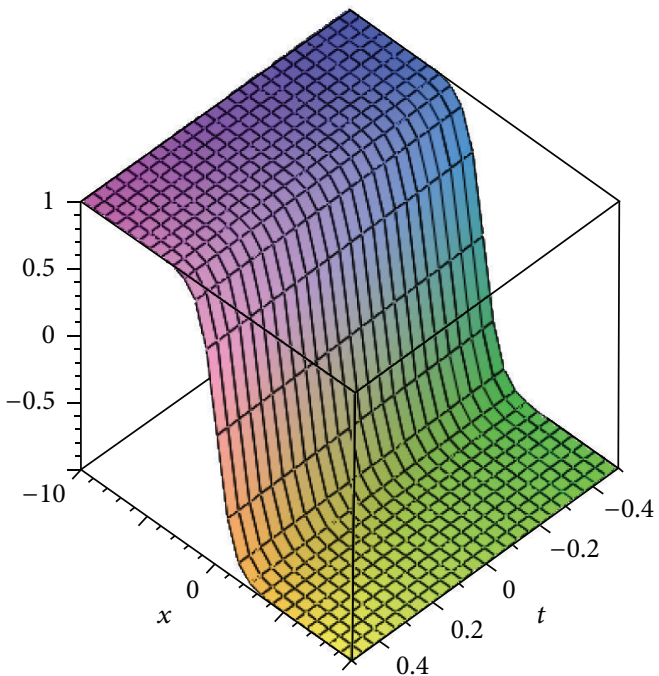

The exact solution for $\phi(x, y, t)$

(a)

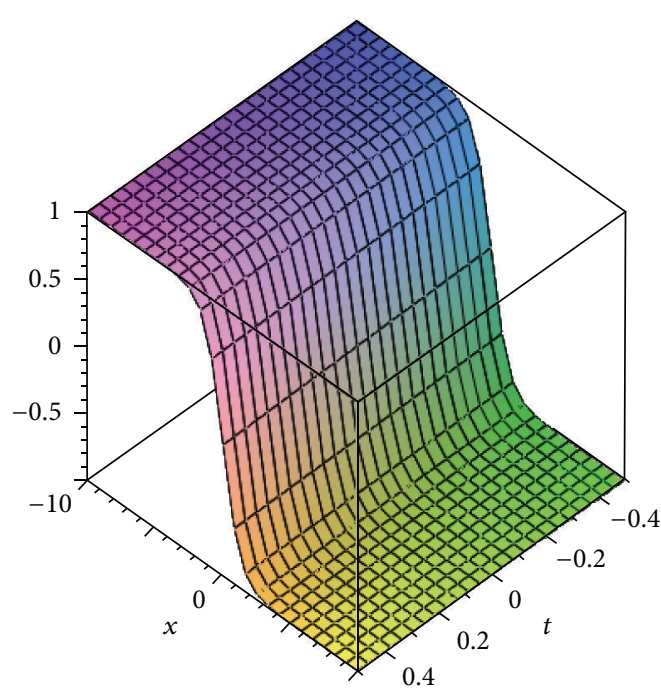

HPM solution for $\phi$ when $q=\rho e^{-0.1 i \theta}$

(c)

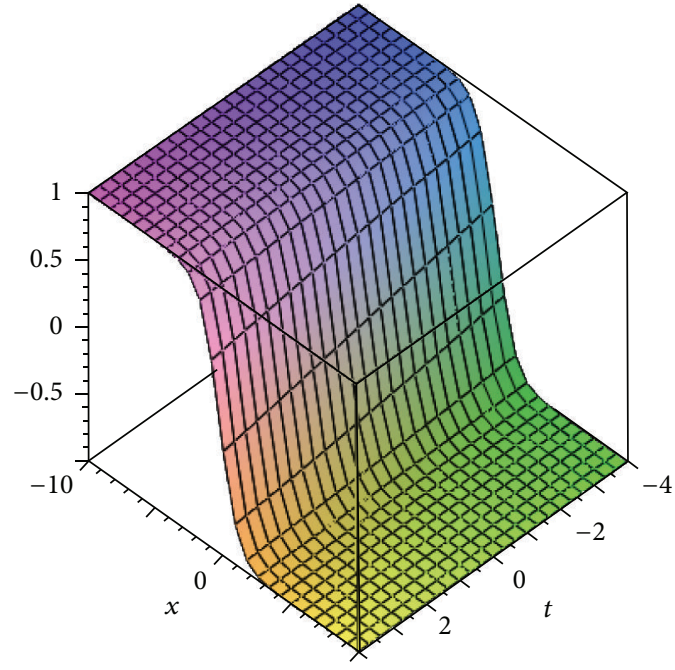

HAM solution for $\phi$ when $q=u+i v$

(b)

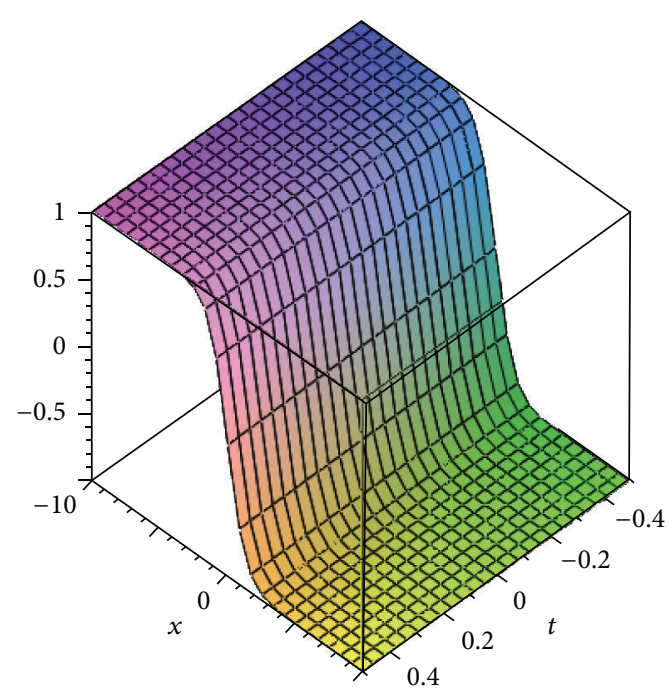

HAM solution for $\phi$ when $q=\rho e^{-0.1 i \theta}$

(d)

Figure 2: Comparison between the exact solution, the HPM solution, and the HAM solution for $\phi(x, y, t)$.

$$
\begin{aligned}
0=( & 2 m v_{1,0} v_{2,0 t}+2 v_{1,0} v_{3,0 x}+\sigma^{2} v_{1,0 x x} \\
+ & 2 \lambda v_{1,0}^{3}-m^{2} \sigma^{2} v_{1,0} v_{2,0 x}^{2} \\
- & \left.m^{2} v_{1,0} v_{2,0 y}^{2}+v_{1,0 y y}+v_{1,1 y y}\right) p \\
+ & \left(2 v_{1,1} v_{3,0 x}+v_{1,2 y y}+2 m v_{1,0} v_{2,1 t}\right. \\
+ & \sigma^{2} v_{1,1 x x}+6 \lambda v_{1,0}^{2} v_{1,1} \\
& -2 m^{2} v_{1,0} v_{2,0 y} v_{2,1 y}-2 m^{2} \sigma^{2} v_{1,0} v_{2,0 x} v_{2,1 x} \\
+ & 2 v_{1,0} v_{3,1 x}+2 m v_{1,1} v_{2,0 t} \\
& \left.-m^{2} \sigma^{2} v_{1,1} v_{2,0 x}^{2}-m^{2} v_{1,1} v_{2,0 y}^{2}\right) p^{2}
\end{aligned}
$$

$$
\begin{aligned}
+ & \left(6 \lambda v_{1,0}^{2} v_{1,2}+v_{1,3 y y}-2 m^{2} \sigma^{2} v_{1,1} v_{2,0 x} v_{2,1 x}\right. \\
+ & 2 m v_{1,0} v_{2,2 t}+2 v_{1,1} v_{3,1 x} \\
+ & 2 m v_{1,2} v_{2,0 t}-m^{2} v_{1,0} v_{2,1 y}^{2} \\
+ & 2 v_{1,2} v_{3,0 x}+\sigma^{2} v_{1,2 x x} \\
& -m^{2} \sigma^{2} v_{1,0} v_{2,1 x}^{2}+2 m v_{1,1} v_{2,1 t} \\
& -m^{2} \sigma^{2} v_{1,2} v_{2,0 x}^{2}-2 m^{2} v_{1,1} v_{2,0 y} v_{2,1 y} \\
& +6 \lambda v_{1,0} v_{1,1}^{2}+2 v_{1,0} v_{3,2 x} \\
& -2 m^{2} v_{1,0} v_{2,0 y} v_{2,2 y}-2 m^{2} \sigma^{2} v_{1,0} v_{2,0 x} v_{2,2 x}
\end{aligned}
$$




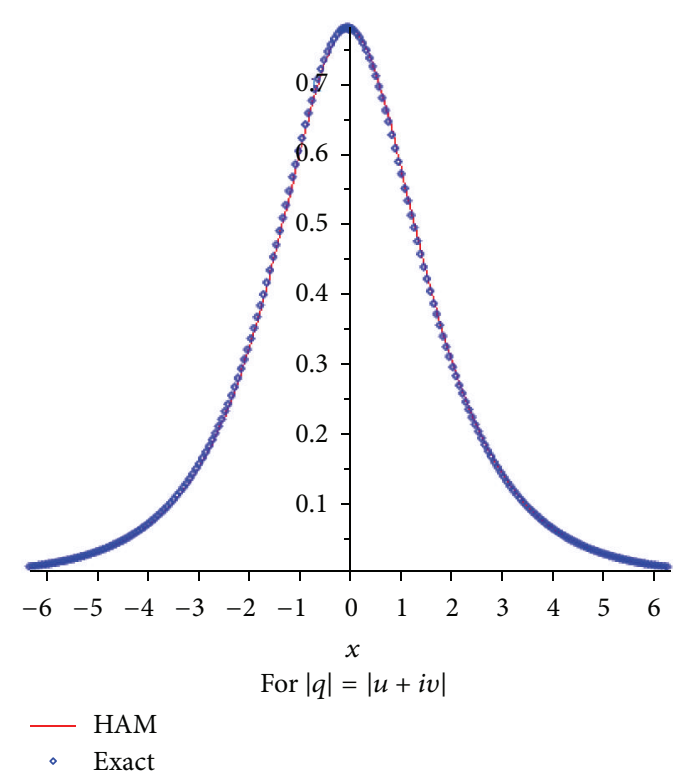

(a)

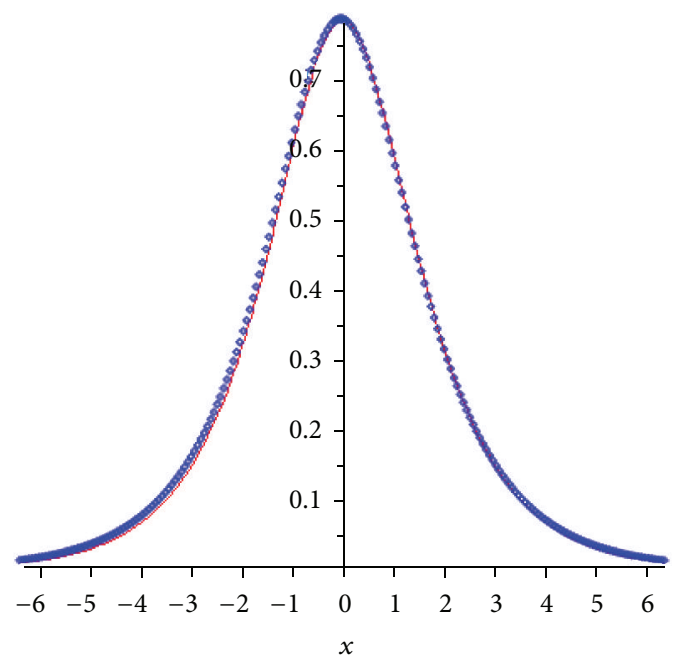

- HPM

For $|q|=\left|\rho e^{-0.1 i \theta}\right|$

- Exact

(b)

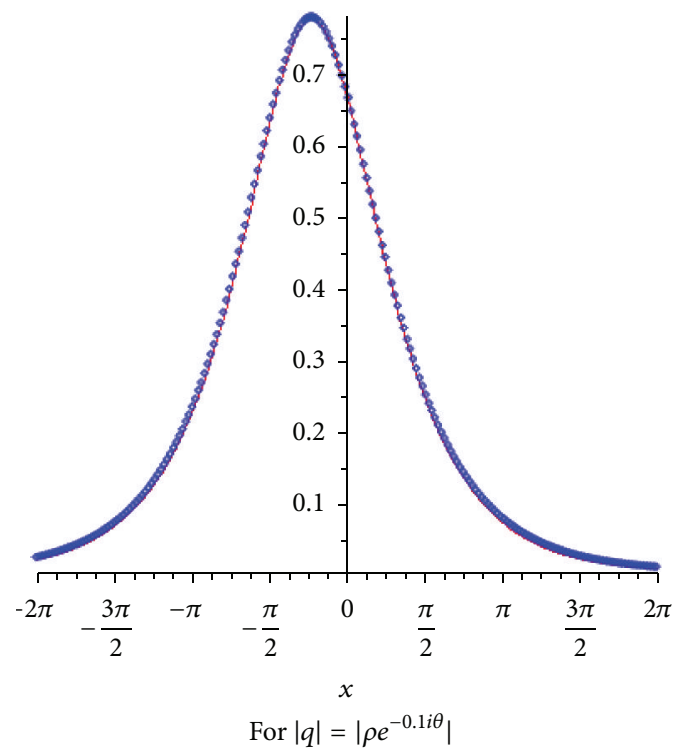

(c)

FIGURE 3: The results obtained by HPM and HAM for $q(x, y, t)$, at $t=0.2$ in comparison with the exact solutions.

$$
\begin{gathered}
\left.-m^{2} v_{1,2} v_{2,0 y}^{2}\right) p^{3}+\cdots \\
0=\left(\sigma^{2} v_{3,1 y y}+\sigma^{2} v_{3,0 y y}-v_{3,0 x x}+4 \lambda v_{1,0} v_{1,0 x}\right) p \\
+\left(\sigma^{2} v_{3,2 y y}-v_{3,1 x x}+4 \lambda v_{1,0} v_{1,1 x}+4 \lambda v_{1,1} v_{1,0 x}\right) p^{2} \\
+\left(\sigma^{2} v_{3,3 y y}-v_{3,2 x x}+4 \lambda v_{1,0} v_{1,2 x}+4 \lambda v_{1,1} v_{1,1 x}\right. \\
\left.+4 \lambda v_{1,2} v_{1,0 x}\right) p^{3}+\cdots
\end{gathered}
$$

To obtain the unknowns $v_{i, j}(x, y, t), i, j=1,2,3$, we must construct and solve the following system which includes nine equations with nine unknowns, considering the initial conditions of $v_{i, j}(x, 0, t)=0, i, j=1,2,3$ :

$$
\begin{aligned}
& -2 v_{1,0 t}+m v_{1,0} v_{2,1 y y}+2 \sigma^{2} m v_{2,0 x} v_{1,0 x} \\
& +\sigma^{2} m v_{2,0 x x} v_{1,0}+m v_{2,0 y y} v_{1,0}+2 m v_{2,0 y} v_{1,0 y}=0 \\
& \sigma^{2} m v_{2,0 x x} v_{1,1}+2 \sigma^{2} m v_{2,1 x} v_{1,0 x}+\sigma^{2} m v_{2,1 x x} v_{1,0} \\
& +m v_{2,1 y y} v_{1,1}-2 v_{1,1 t}+m v_{2,0 y y} v_{1,1} \\
& +2 \sigma^{2} m v_{2,0 x} v_{1,1 x}+m v_{2,2 y y} v_{1,0}+2 m v_{2,0 y} v_{1,1 y} \\
& +2 m v_{2,1 y} v_{1,0 y}=0
\end{aligned}
$$




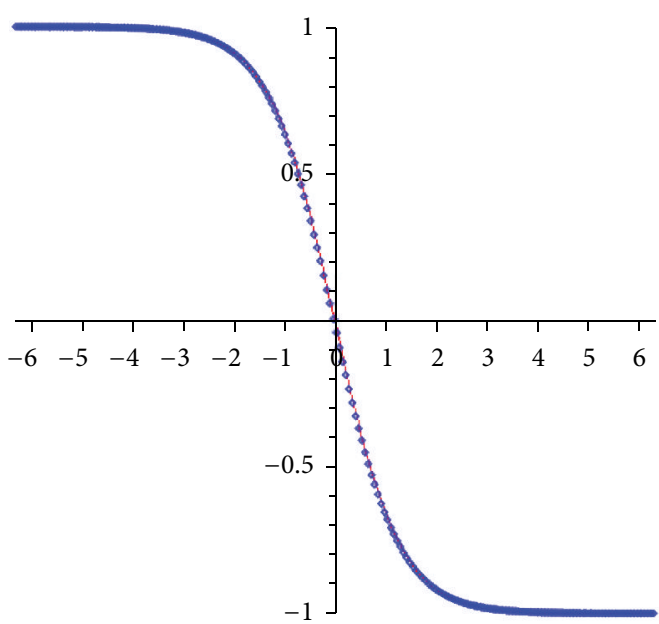

When $q=u+i v$

- HAM

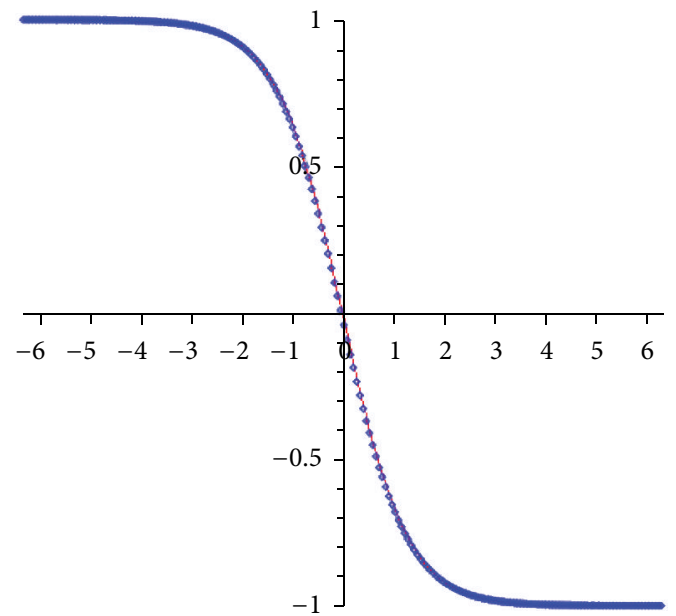

When $q=\rho e^{-0.1 i \theta}$

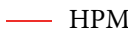

- Exact

(a)

(b)

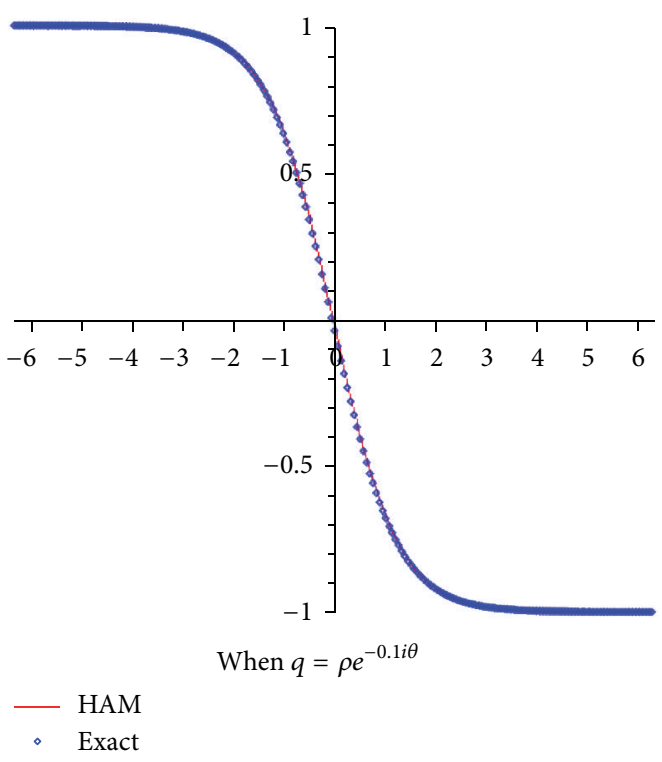

(c)

FIgURE 4: The results obtained by HPM and HAM for $\phi(x, y, t)$, at $t=0.2$ in comparison with the exact solutions.

$$
\begin{aligned}
& 2 m v_{2,2 y} v_{1,0 y}+2 \sigma^{2} m v_{2,0 x} v_{1,2 x}+2 \sigma^{2} m v_{2,1 x} v_{1,1 x} \\
& +m v_{2,1 y y} v_{1,2}-2 v_{1,2 t}+m v_{2,0 y y} v_{1,2}+\sigma^{2} m v_{2,0 x x} v_{1,2} \\
& +\sigma^{2} m v_{2,1 x x} v_{1,1}+m v_{2,3 y y} v_{1,0}+v_{2,2 y y} m v_{1,1} \\
& +2 m v_{2,0 y} v_{1,2 y}+2 \sigma^{2} m v_{2,2 x} v_{1,0 x}+2 m v_{2,1 y} v_{1,1 y} \\
& +\sigma^{2} m v_{2,2 x x} v_{1,0}=0 \\
& 2 m v_{1,0} v_{2,0 t}+2 v_{1,0} v_{3,0 x}+\sigma^{2} v_{1,0 x x}+2 \lambda v_{1,0}^{3} \\
& \quad-m^{2} \sigma^{2} v_{1,0} v_{2,0 x}^{2}-m^{2} v_{1,0} v_{2,0 y}^{2}+v_{1,0 y y}+v_{1,1 y y}=0
\end{aligned}
$$

$$
\begin{aligned}
& 2 v_{1,1} v_{3,0 x}+v_{1,2 y y}+2 m v_{1,0} v_{2,1 t} \\
& +\sigma^{2} v_{1,1 x x}+6 \lambda v_{1,0}^{2} v_{1,1}-2 m^{2} v_{1,0} v_{2,0 y} v_{2,1 y} \\
& -2 m^{2} \sigma^{2} v_{1,0} v_{2,0 x} v_{2,1 x}+2 v_{1,0} v_{3,1 x}+2 m v_{1,1} v_{2,0 t} \\
& -m^{2} \sigma^{2} v_{1,1} v_{2,0 x}^{2}-m^{2} v_{1,1} v_{2,0 y}^{2}=0 \\
& 6 \lambda v_{1,0}^{2} v_{1,2}+v_{1,3 y y}-2 m^{2} \sigma^{2} v_{1,1} v_{2,0 x} v_{2,1 x} \\
& +2 m v_{1,0} v_{2,2 t}+2 v_{1,1} v_{3,1 x}+2 m v_{1,2} v_{2,0 t} \\
& \quad-m^{2} v_{1,0} v_{2,1 y}^{2}+2 v_{1,2} v_{3,0 x}+\sigma^{2} v_{1,2 x x}
\end{aligned}
$$




$$
\begin{gathered}
-m^{2} \sigma^{2} v_{1,0} v_{2,1 x}^{2}+2 m v_{1,1} v_{2,1 t}-m^{2} \sigma^{2} v_{1,2} v_{2,0 x}^{2} \\
-2 m^{2} v_{1,1} v_{2,0 y} v_{2,1 y}+6 \lambda v_{1,0} v_{1,1}^{2}+2 v_{1,0} v_{3,2 x} \\
-2 m^{2} v_{1,0} v_{2,0 y} v_{2,2 y}-2 m^{2} \sigma^{2} v_{1,0} v_{2,0 x} v_{2,2 x} \\
-m^{2} v_{1,2} v_{2,0 y}^{2}=0 \\
\sigma^{2} v_{3,1 y y}+\sigma^{2} v_{3,0 y y}-v_{3,0 x x}+4 \lambda v_{1,0} v_{1,0 x}=0 \\
\sigma^{2} v_{3,2 y y}-v_{3,1 x x}+4 \lambda v_{1,0} v_{1,1 x}+4 \lambda v_{1,1} v_{1,0 x}=0 \\
\sigma^{2} v_{3,3 y y}-v_{3,2 x x}+4 \lambda v_{1,0} v_{1,2 x}+4 \lambda v_{1,1} v_{1,1 x} \\
+4 \lambda v_{1,2} v_{1,0 x}=0
\end{gathered}
$$

From (8), if the three approximations are sufficient, we will obtain

$$
\begin{aligned}
& \rho(x, y, t)=\lim _{p \rightarrow 1} V_{1}(x, y, t)=\sum_{k=0}^{3} v_{1, k}(x, y, t), \\
& \theta(x, y, t)=\lim _{p \rightarrow 1} V_{2}(x, y, t)=\sum_{k=0}^{3} v_{2, k}(x, y, t), \\
& \phi(x, y, t)=\lim _{p \rightarrow 1} V_{3}(x, y, t)=\sum_{k=0}^{3} v_{3, k}(x, y, t) .
\end{aligned}
$$

To calculate the terms of the homotopy series (19) for $\rho(x, y, t), \theta(x, y, t)$, and $\phi(x, y, t)$, we substitute the initial conditions (5) into the system (9), and using Mathematica software, from (13), we obtain

$$
\begin{aligned}
& v_{1,1} \frac{1}{2} r y^{2} \operatorname{sech}[s(-c t+x)] \\
& \times\left(\cos \left[k_{3} t+k_{1} x\right]\right. \\
& \times\left(k_{1}^{2} \sigma^{2}-2 k_{3} m r \cos \left[k_{3} t+k_{1} x\right]\right. \\
& \times \operatorname{sech}[s(-c t+x)] \\
& +\left(s\left(-2 f+s \sigma^{2}\right)\right. \\
& \quad+r^{2}\left(-2 \lambda+k_{1}^{2} m^{2} \sigma^{2}\right) \\
& \left.\quad \times \cos ^{2}\left[k_{3} t+k_{1} x\right]\right) \\
& \left.\times\left(\operatorname{sech}{ }^{2}[s(-c t+x)]\right)\right) \\
& -s\left(2 k_{1} \sigma^{2} \sin \left[k_{3} t+k_{1} x\right]+m r \operatorname{sech}[s(-c t+x)]\right. \\
& \quad \times\left(c+k_{1} m r \sigma^{2} \cos \left[k_{3} t+k_{1} x\right] \operatorname{sech}[s(-c t+x)]\right) \\
& \left.\quad \times \sin \left[2\left(k_{3} t+k_{1} x\right)\right]\right) \tanh [s(-c t+x)]
\end{aligned}
$$

$$
\begin{aligned}
& +s^{2} \sigma^{2} \cos \left[k_{3} t+k_{1} x\right] \\
& \times\left(-1+m^{2} r^{2} \operatorname{sech}^{2}[s(-c t+x)]\left(\sin ^{2}\left[k_{3} t+k_{1} x\right]\right)\right) \\
& \left.\times \tanh ^{2}[s(-c t+x)]\right) .
\end{aligned}
$$

From (10), we obtain

$$
\begin{aligned}
& v_{2,1} \\
& \qquad \begin{array}{l}
\frac{1}{2 m} y^{2}\left(m r s^{2} \sigma^{2} \operatorname{sech}^{3}[s(-c t+x)] \sin \left[k_{3} t+k_{1} x\right]\right. \\
-2 k_{3} \tan \left[k_{3} t+k_{1} x\right]+2 c s \tanh [s(-c t+x)] \\
+m r \sigma^{2} \operatorname{sech}[s(-c t+x)] \\
\times\left(2 k_{1}^{2} \sin \left[k_{3} t+k_{1} x\right]+s \tanh [s(-c t+x)]\right. \\
\times\left(k_{1}\left(1+3 \cos \left[2\left(k_{3} t+k_{1} x\right)\right]\right)\right. \\
\quad \times \sec \left[k_{3} t+k_{1} x\right]-3 s \sin \left[k_{3} t+k_{1} x\right] \\
\times \tanh [s(-c t+x)]))) .
\end{array}
\end{aligned}
$$

From (16), we obtain

$$
\begin{aligned}
& v_{3,1} \\
& =\frac{1}{\sigma^{2}}\left(y ^ { 2 } \operatorname { s e c h } ^ { 2 } [ s ( - c t + x ) ] \left(k_{1} r^{2} \lambda \sin \left[2\left(k_{3} t+k_{1} x\right)\right]\right.\right. \\
& \left.+s\left(-f_{s}+2 r^{2} \lambda \cos ^{2}\left[k_{3} t+k_{1} x\right]\right) \tanh [s(-c t+x)]\right) .
\end{aligned}
$$

In this manner, the other components $v_{1,2}(x, y, t)$, $v_{2,2}(x, y, t), \quad v_{3,2}(x, y, t), \quad v_{1,3}(x, y, t), \quad v_{2,3}(x, y, t), \quad$ and $v_{3,3}(x, y, t)$ can be obtained from (14), (11), (17), (15), (12), and (18), respectively, and substituting these components into (19) to obtain $\rho(x, y, t), \theta(x, y, t)$, and $\phi(x, y, t)$.

\section{Application of the Homotopy Analysis Method}

In order to apply the homotopy analysis method for (2), we choose the linear operator $L\left[\varphi_{i}(x, y, t ; p)\right]=\partial^{2} \varphi_{i} / \partial y^{2}$ with the property $L\left[c_{1, i}+c_{2, i} y\right]=0, i=1,2,3$, where $c_{1, i}, c_{2, i}$ are integral constants to be determined by initial conditions.

Furthermore, (2) suggests to define the nonlinear operators

$$
\begin{aligned}
& N_{1}=\sigma^{4} \frac{\partial^{2} \varphi_{2}}{\partial y^{2}}+\sigma^{2} \frac{\partial^{2} \varphi_{2}}{\partial x^{2}}+2 \frac{\partial \varphi_{1}}{\partial t}-2 \varphi_{2} \frac{\partial \varphi_{3}}{\partial x}+2 \lambda\left(\varphi_{1}^{2}+\varphi_{2}^{2}\right) \varphi_{2} \\
& N_{2}=\sigma^{4} \frac{\partial^{2} \varphi_{1}}{\partial y^{2}}+\sigma^{2} \frac{\partial^{2} \varphi_{1}}{\partial x^{2}}-2 \frac{\partial \varphi_{2}}{\partial t}-2 \varphi_{1} \frac{\partial \varphi_{3}}{\partial x}+2 \lambda\left(\varphi_{1}^{2}+\varphi_{2}^{2}\right) \varphi_{1} \\
& N_{3}=\frac{\partial^{2} \varphi_{3}}{\partial y^{2}}-\frac{1}{\sigma^{2}} \frac{\partial^{2} \varphi_{3}}{\partial x^{2}}+\frac{2 \lambda}{\sigma^{2}} \frac{\partial\left(\varphi_{1}^{2}+\varphi_{2}^{2}\right)}{\partial x}
\end{aligned}
$$


we construct the zero-order deformation equations

$$
\begin{aligned}
& (1-p) L\left[\varphi_{i}(x, y, t ; p)-z_{i, 0}(x, y, t)\right] \\
& =p \hbar_{i} N_{i}\left[\varphi_{i}(x, y, t ; p)\right], \quad i=1,2,3 .
\end{aligned}
$$

When $p=0$

$$
\begin{aligned}
\varphi_{1}(x, y, t ; 0) & =z_{1,0}(x, y, t)=u_{0}(x, y, t) \\
& =r \operatorname{sech}[s(x-c t)] \cos \left[k_{1} x+k_{3} t\right], \\
\varphi_{2}(x, y, t ; 0) & =z_{2,0}(x, y, t)=v_{0}(x, y, t) \\
& =r \operatorname{sech}[s(x-c t)] \sin \left[k_{1} x+k_{3} t\right], \\
\varphi_{3}(x, y, t ; 0) & =z_{3,0}(x, y, t)=\phi_{0}(x, y, t) \\
& =f \tanh [s(x-c t)] .
\end{aligned}
$$

When $p=1$

$$
\begin{aligned}
& \varphi_{1}(x, y, t ; 1)=u(x, y, t), \\
& \varphi_{2}(x, y, t ; 1)=v(x, y, t), \\
& \varphi_{3}(x, y, t ; 1)=\phi(x, y, t) .
\end{aligned}
$$

Therefore, as the embedding parameter $p$ increases from 0 to $1, \varphi_{i}(x, y, t ; p)$ varies from initial guesses $z_{i, 0}(x, y, t)$ to the solutions $u(x, y, t), v(x, y, t)$ and $\phi(x, y, t)$, for $i=1,2,3$, respectively.

Expanding $\varphi_{i}(x, y, t ; p)$ in Taylor series with respect to $p$ for $i=1,2,3$, one has

$$
\varphi_{i}(x, y, t ; p)=z_{i, 0}(x, y, t)+\sum_{m=1}^{+\infty} z_{i, m}(x, y, t) p^{m}
$$

where

$$
z_{i, m}(x, y, t)=\left.\frac{1}{m !} \frac{\partial^{m} \varphi_{i}(x, y, t ; p)}{\partial p^{m}}\right|_{p=0} .
$$

If the auxiliary linear operator, the initial guesses, and the auxiliary parameters $\hbar_{i}$ are so properly chosen, the above series converge at $p=1$, and

$$
\begin{aligned}
& u(x, y, t)=z_{1,0}(x, y, t)+\sum_{m=1}^{+\infty} z_{1, m}(x, y, t), \\
& v(x, y, t)=z_{2,0}(x, y, t)+\sum_{m=1}^{+\infty} z_{2, m}(x, y, t), \\
& \phi(x, y, t)=z_{3,0}(x, y, t)+\sum_{m=1}^{+\infty} z_{3, m}(x, y, t),
\end{aligned}
$$

which must be one of solutions of the original nonlinear equations as proved by Liao [13]. Define the vectors

$$
\begin{array}{r}
\vec{z}_{i, n}=\left\{z_{i, 0}(x, y, t), z_{i, 1}(x, y, t), \ldots, z_{i, n}(x, y, t)\right\} \\
i=1,2,3 .
\end{array}
$$

We have the $m$ th-order deformation equations

$$
\begin{aligned}
L & {\left[z_{i, m}(x, y, t)-\chi_{m} z_{i, m-1}(x, y, t)\right] } \\
& =\hbar_{i} R_{i, m}\left(\vec{z}_{1, m-1}, \vec{z}_{2, m-1}, \vec{z}_{3, m-1}\right), \quad i=1,2,3,
\end{aligned}
$$

where

$$
\begin{aligned}
R_{1, m} & \left(\vec{z}_{1, m-1}, \vec{z}_{2, m-1}, \vec{z}_{3, m-1}\right) \\
= & \sigma^{4} \frac{\partial^{2} z_{2, m-1}}{\partial y^{2}}+\sigma^{2} \frac{\partial^{2} z_{2, m-1}}{\partial x^{2}}+2 \frac{\partial z_{1, m-1}}{\partial t} \\
& -2 \sum_{n=0}^{m-1} z_{2, n} \frac{\partial z_{3, m-1-n}}{\partial x} \\
& +2 \lambda \sum_{n=0}^{m-1} \sum_{k=0}^{n}\left(z_{1, k} z_{1, n-k}+z_{2, k} z_{2, n-k}\right) z_{2, m-1-n} \\
R_{2, m} & \left(\vec{z}_{1, m-1}, \vec{z}_{2, m-1}, \vec{z}_{3, m-1}\right) \\
= & \sigma^{4} \frac{\partial^{2} z_{1, m-1}}{\partial y^{2}}+\sigma^{2} \frac{\partial^{2} z_{1, m-1}}{\partial x^{2}}-2 \frac{\partial z_{2, m-1}}{\partial t} \\
& -2 \sum_{n=0}^{m-1} z_{1, n} \frac{\partial z_{3, m-1-n}}{\partial x} \\
& +\frac{2 \lambda}{\sigma^{2}} \frac{\partial}{\partial x} \sum_{n=0}^{m-1}\left(z_{1, n} z_{1, m-1-n}+z_{2, n} z_{2, m-1-n}\right) \\
& +2 \lambda \sum_{n=0}^{m-1} \sum_{k=0}^{n}\left(z_{1, k} z_{1, n-k}+z_{2, k} z_{2, n-k}\right) z_{1, m-1-n} \\
R_{3, m} & \left(\vec{z}_{1, m-1}, \vec{z}_{2, m-1}, \vec{z}_{3, m-1}\right) \\
& \frac{\partial y_{3, m-1}^{2}-\frac{1}{\sigma^{2}} \frac{\partial^{2} z_{3, m-1}}{\partial x^{2}}}{\partial y^{2}} \\
& \\
&
\end{aligned}
$$

where $z_{1}, z_{2}$, and $z_{3}$ are functions of $x, y$, and $t$, and

$$
\chi_{m}= \begin{cases}0, & m \leq 1 \\ 1, & m>1\end{cases}
$$

Now, the solutions of the $m$ th-order deformation (31) for $m \geq$ 1 become

$$
\begin{aligned}
z_{i, m}(x, y, t)= & \chi_{m} z_{i, m-1}(x, y, t) \\
& +\hbar_{i} \iint_{0}^{y}\left[R_{i, m}\left(\vec{z}_{1, m-1}, \vec{z}_{2, m-1}, \vec{z}_{3, m-1}\right)\right] d y d y \\
& +c_{1, i}+c_{2, i} y
\end{aligned}
$$

For simplicity, we suppose $\hbar_{1}=\hbar_{2}=\hbar_{3}=\hbar$. 
We consider the solutions of (2) with the initial conditions (25). We now obtain at $m=1$

$$
\begin{aligned}
& z_{1,1}(x, y, t)=\frac{1}{2} \hbar r y^{2} \operatorname{sech}[s(x-c t)] \\
& \times\left\{-\left[2 k_{3}+k_{1}^{2} \sigma^{2}+\left(2 f s-2 r^{2} \lambda+s^{2} \sigma^{2}\right)\right.\right. \\
& \left.\times \operatorname{sech}^{2}[s(x-c t)]\right] \cos \left[k_{1} x+k_{3} t\right] \\
& -2 s\left(c-k_{1} \sigma^{2}\right) \sin \left[k_{1} x+k_{3} t\right] \\
& \times \tanh [s(x-c t)]+s^{2} \sigma^{2} \\
& \left.\times \cos \left[k_{1} x+k_{3} t\right] \tanh ^{2}[s(x-c t)]\right\}, \\
& z_{2,1}(x, y, t)=\frac{1}{2} \hbar r y^{2} \operatorname{sech}[s(x-c t)] \\
& \times\left\{-\left[2 k_{3}+k_{1}^{2} \sigma^{2}+\left(2 f s-2 r^{2} \lambda+s^{2} \sigma^{2}\right)\right.\right. \\
& \left.\times \operatorname{sech}^{2}[s(x-c t)]\right] \sin \left[k_{1} x+k_{3} t\right] \\
& +2 s\left(c-k_{1} \sigma^{2}\right) \cos \left[k_{1} x+k_{3} t\right] \\
& \times \tanh [s(x-c t)] \\
& +s^{2} \sigma^{2} \sin \left[k_{1} x+k_{3} t\right] \\
& \left.\times \tanh ^{2}[s(x-c t)]\right\}, \\
& z_{3,1}(x, y, t)=\frac{1}{\sigma^{2}}\left(\hbar s y^{2}\left(f s-2 r^{2} \lambda\right) \operatorname{sech}^{2}\right. \\
& \times[s(x-c t)] \tanh [s(x-c t)]) .
\end{aligned}
$$

Obviously, for $\hbar=-1$, the obtained solutions are the same homotopy perturbation method in [2]; we continue to evaluate two terms of HAM.

Now for (4), we choose the linear operator $L\left[\varphi_{i}(x, y, t ; p)\right]=\partial^{2} \varphi_{i} / \partial y^{2}$ with the property $\left[c_{1, i}+c_{2, i} y\right]=0$, $i=1,2,3$, where $c_{1, i}, c_{2, i}$ are integral constant to be determined by initial conditions.

Furthermore, (4) suggests to define the nonlinear operators

$$
\begin{aligned}
N_{1}= & \varphi_{1} \frac{\partial^{2} \varphi_{2}}{\partial y^{2}}+2 \frac{\partial \varphi_{2}}{\partial y} \frac{\partial \varphi_{1}}{\partial y}+2 \sigma^{2} \frac{\partial \varphi_{2}}{\partial x} \frac{\partial \varphi_{1}}{\partial x} \\
& +\sigma^{2} \varphi_{1} \frac{\partial^{2} \varphi_{2}}{\partial x^{2}}-\frac{2}{m} \frac{\partial \varphi_{1}}{\partial t} \\
N_{2}= & \frac{\partial^{2} \varphi_{1}}{\partial y^{2}}-m^{2} \varphi_{1}\left(\left(\frac{\partial \varphi_{2}}{\partial y}\right)^{2}+\sigma^{2}\left(\frac{\partial \varphi_{2}}{\partial x}\right)^{2}\right) \\
& +\sigma^{2} \frac{\partial^{2} \varphi_{1}}{\partial x^{2}}+2 m \varphi_{1} \frac{\partial \varphi_{2}}{\partial t}+2 \lambda \varphi_{1}^{3}+2 \varphi_{1} \frac{\partial \varphi_{3}}{\partial x} \\
N_{3}= & \frac{\partial^{2} \varphi_{3}}{\partial y^{2}}-\frac{1}{\sigma^{2}} \frac{\partial^{2} \varphi_{3}}{\partial x^{2}}+\frac{4 \lambda}{\sigma^{2}} \varphi_{1} \frac{\partial \varphi_{1}}{\partial x}
\end{aligned}
$$

We construct the zero-order deformation equations

$$
\begin{aligned}
& (1-p) L\left[\varphi_{i}(x, y, t ; p)-z_{i, 0}(x, y, t)\right] \\
& =p \hbar_{i} N_{i}\left[\varphi_{i}(x, y, t ; p)\right], \quad i=1,2,3 .
\end{aligned}
$$

When $p=0$,

$$
\begin{aligned}
\varphi_{1}(x, y, t ; 0) & =z_{1,0}(x, y, t)=\rho_{0}(x, y, t) \\
& =r \operatorname{sech}[s(x-c t)] \cos \left[k_{1} x+k_{3} t\right], \\
\varphi_{2}(x, y, t ; 0) & =z_{2,0}(x, y, t)=\theta_{0}(x, y, t) \\
& =r \operatorname{sech}[s(x-c t)] \sin \left[k_{1} x+k_{3} t\right], \\
\varphi_{3}(x, y, t ; 0) & =z_{3,0}(x, y, t)=\phi_{0}(x, y, t) \\
& =f \tanh [s(x-c t)] .
\end{aligned}
$$

When $p=1$,

$$
\begin{aligned}
& \varphi_{1}(x, y, t ; 1)=\rho(x, y, t), \\
& \varphi_{2}(x, y, t ; 1)=\theta(x, y, t), \\
& \varphi_{3}(x, y, t ; 1)=\phi(x, y, t) .
\end{aligned}
$$

Therefore, as the embedding parameter $p$ increases from 0 to $1, \varphi_{i}(x, y, t ; p)$ varies from initial guesses $z_{i, 0}(x, y, t)$ to the solutions $\rho(x, y, t), \theta(x, y, t)$, and $\phi(x, y, t)$, for $i=1,2,3$, respectively.

Expanding $\varphi_{i}(x, y, t ; p)$ in Taylor series with respect to $p$ for $i=1,2,3$, one has

$$
\varphi_{i}(x, y, t ; p)=z_{i, 0}(x, y, t)+\sum_{m=1}^{+\infty} z_{i, m}(x, y, t) p^{m},
$$

where

$$
z_{i, m}(x, y, t)=\left.\frac{1}{m !} \frac{\partial^{m} \varphi_{i}(x, y, t ; p)}{\partial p^{m}}\right|_{p=0}
$$

If the auxiliary linear operator, the initial guesses, and the auxiliary parameters $\hbar_{i}$ are so properly chosen, the series (40) converge at $p=1$, has

$$
\begin{aligned}
& \rho(x, y, t)=z_{1,0}(x, y, t)+\sum_{m=1}^{+\infty} z_{1, m}(x, y, t), \\
& \theta(x, y, t)=z_{2,0}(x, y, t)+\sum_{m=1}^{+\infty} z_{2, m}(x, y, t), \\
& \phi(x, y, t)=z_{3,0}(x, y, t)+\sum_{m=1}^{+\infty} z_{3, m}(x, y, t),
\end{aligned}
$$

which must be one of solutions of the original nonlinear equation as proved by Liao [13]. Define the vectors

$$
\begin{array}{r}
\vec{z}_{i, n}=\left\{z_{i, 0}(x, y, t), z_{i, 1}(x, y, t), \ldots, z_{i, n}(x, y, t)\right\} \\
i=1,2,3 .
\end{array}
$$


We have the $m$ th-order deformation equations

$$
\begin{aligned}
L & {\left[z_{i, m}(x, y, t)-\chi_{m} z_{i, m-1}(x, y, t)\right] } \\
& =\hbar_{i} R_{i, m}\left(\vec{z}_{1, m-1}, \vec{z}_{2, m-1}, \vec{z}_{3, m-1}\right), \quad i=1,2,3,
\end{aligned}
$$

where

$$
\begin{aligned}
& R_{1, m}\left(\vec{z}_{1, m-1}, \vec{z}_{2, m-1}, \vec{z}_{3, m-1}\right) \\
&= \sum_{n=0}^{m-1} z_{1, n} \frac{\partial^{2} z_{2, m-1-n}}{\partial y^{2}}+2 \sum_{n=0}^{m-1} \frac{\partial z_{2, n}}{\partial y} \frac{\partial z_{1, m-1-n}}{\partial y} \\
&+ 2 \sigma^{2} \sum_{n=0}^{m-1} \frac{\partial z_{2, n}}{\partial x} \frac{\partial z_{1, m-1-n}}{\partial x}-\frac{2}{m} \frac{\partial z_{1, m-1}}{\partial t} \\
&+ \sigma^{2} \sum_{n=0}^{m-1} z_{1, n} \frac{\partial^{2} z_{2, m-1-n}}{\partial x^{2}}, \\
& R_{2, m}\left(\vec{z}_{1, m-1}, \vec{z}_{2, m-1}, \vec{z}_{3, m-1}\right) \\
&=\frac{\partial^{2} z_{1, m-1}}{\partial y^{2}}-m^{2} \sum_{n=0}^{m-1} \sum_{k=0}^{n} z_{1, k} \frac{\partial z_{2, n-k} \frac{\partial z_{2, m-1-n}}{\partial y}}{\partial y} \\
& R_{3, m}\left(\vec{z}_{1, m-1}, \vec{z}_{2, m-1}, \vec{z}_{3, m-1}\right) \\
& \quad \frac{\partial^{2} z_{3, m-1}}{\partial y^{2}}-\frac{1}{\sigma^{2}} \frac{\partial^{2} z_{3, m-1}}{\partial x^{2}}+\frac{4 \lambda}{\sigma^{2}} \sum_{n=0}^{m-1} z_{1, n} \frac{\partial z_{1, m-1-n}}{\partial x} \\
&+m^{2} \sigma^{2} \sum_{n=0}^{m-1} \sum_{k=0}^{n} z_{1, k} \frac{\partial z_{2, n-k}}{\partial x} \frac{\partial z_{2, m-1-n}}{\partial x} \\
& \frac{\partial_{1, m-1}}{\partial x^{2}}+2 \lambda \sum_{n=0}^{m-1} \sum_{k=0}^{n} z_{1, k} z_{1, n-k} z_{1, m-1-n} \\
& \frac{\partial z_{3, m-1-n}}{\partial x}+2 m \sum_{n=0}^{m-1} z_{1, n} \frac{\partial z_{2, m-1-n}}{\partial t}
\end{aligned}
$$

where $z_{1}, z_{2}$, and $z_{3}$ are functions of $x, y$, and $t$, and

$$
\chi_{m}= \begin{cases}0, & m \leq 1 \\ 1, & m>1\end{cases}
$$

Now, the solutions of the $m$ th-order deformation (44) for $m \geq 1$ become

$$
\begin{aligned}
z_{i, m}(x, y, t)= & \chi_{m} z_{i, m-1}(x, y, t) \\
& +\hbar_{i} \iint_{0}^{y}\left[R_{i, m}\left(\vec{z}_{1, m-1}, \vec{z}_{2, m-1}, \vec{z}_{3, m-1}\right)\right] d y d y \\
& +c_{1, i}+c_{2, i} y .
\end{aligned}
$$

For simplicity, we suppose $\hbar_{1}=\hbar_{2}=\hbar_{3}=\hbar$.
TABLE 1: The HPM results for $q(x, y, t)$ in comparison with the analytical solution with initial conditions (5).

\begin{tabular}{lc}
\hline$t$ & $|q e-q|$ \\
\hline-0.6 & $9.861869456 \times 10^{-18}$ \\
-0.4 & $1.073360934 \times 10^{-17}$ \\
-0.2 & $1.161066111 \times 10^{-17}$ \\
0 & $1.248899278 \times 10^{-17}$ \\
0.2 & $1.336466228 \times 10^{-17}$ \\
0.4 & $1.423383001 \times 10^{-17}$ \\
0.6 & $1.509277063 \times 10^{-17}$ \\
\hline
\end{tabular}

TABLE 2: The HPM results for $\phi(x, y, t)$ in comparison with the analytical solution with initial conditions (5).

\begin{tabular}{lc}
\hline$t$ & $|\phi e-\phi|$ \\
\hline-0.6 & $3.3574 \times 10^{-15}$ \\
-0.4 & $1.9466 \times 10^{-15}$ \\
-0.2 & $1.52954 \times 10^{-15}$ \\
0 & $1.81125 \times 10^{-15}$ \\
0.2 & $2.77765 \times 10^{-15}$ \\
0.4 & $5.04003 \times 10^{-15}$ \\
0.6 & $1.07655 \times 10^{-15}$ \\
\hline
\end{tabular}

TABLE 3: The HAM results for $q(x, y, t)$ in comparison with the analytical solution with initial conditions (3).

\begin{tabular}{lc}
\hline$t$ & $|q e-q|$ \\
\hline-0.6 & $1.415900000 \times 10^{-19}$ \\
-0.4 & $1.400513800 \times 10^{-19}$ \\
-0.2 & $1.385294700 \times 10^{-19}$ \\
0 & $1.370240800 \times 10^{-19}$ \\
0.2 & $1.355350700 \times 10^{-19}$ \\
0.4 & $1.340622400 \times 10^{-19}$ \\
0.6 & $1.326054200 \times 10^{-19}$ \\
\hline
\end{tabular}

We consider the solutions of (4) with the initial conditions (38) and obtain for $m=1$

$$
\begin{aligned}
& z_{1,1}(x, y, t) \\
& =\frac{1}{2} \hbar r y^{2} \operatorname{sech}[s(-c t+x)] \\
& \times\left(\cos \left[k_{3} t+k_{1} x\right]\right. \\
& \quad \times\left(-k_{1}^{2} \sigma^{2}+2 k_{3} m r \cos \left[k_{3} t+k_{1} x\right] \operatorname{sech}[s(-c t+x)]\right. \\
& +\left(s\left(2 f-s \sigma^{2}\right)+r^{2}\left(2 \lambda-k_{1}^{2} m^{2} \sigma^{2}\right)\right. \\
& \left.\left.\quad \times \cos ^{2}\left[k_{3} t+k_{1} x\right]\right) \operatorname{sech}^{2}[s(-c t+x)]\right)
\end{aligned}
$$




$$
\begin{aligned}
& +s\left(2 k_{1} \sigma^{2} \sin \left[k_{3} t+k_{1} x\right]+m r \operatorname{sech}[s(-c t+x)]\right. \\
& \times\left(c+k_{1} m r \sigma^{2} \cos \left[k_{3} t+k_{1} x\right] \operatorname{sech}[s(-c t+x)]\right) \\
& \left.\times \sin \left[2\left(k_{3} t+k_{1} x\right)\right]\right) \tanh [s(-c t+x)] \\
& +s^{2} \sigma^{2} \cos \left[k^{3} t+k^{1} x\right] \\
& \times\left(1-m^{2} r^{2} \operatorname{sech}^{2}[s(-c t+x)] \sin ^{2}\left[k_{3} t+k_{1} x\right]\right) \\
& \left.\times \tanh ^{2}[s(-c t+x)]\right)
\end{aligned}
$$

$$
z_{2,1}(x, y, t)
$$$$
=\frac{1}{2 m} \hbar y^{2}\left(2 k_{3} \tan \left[k_{3} t+k_{1} x\right]+m r \sigma^{2} \operatorname{sech}[s(-c t+x)]\right.
$$$$
\times\left(\left(-3 k_{1}^{2}+3 s^{2}-4 s^{2} \operatorname{sech}^{2}[s(-c t+x)]\right)\right.
$$$$
\times \sin \left[k_{3} t+k_{1} x\right]
$$$$
+k_{1} s\left(1+3 \cos \left[2\left(k_{3} t+k_{1} x\right)\right]\right)
$$$$
\left.\times \sec \left[k_{3} t+k_{1} x\right] \tanh [s(c t-x)]\right)
$$$$
-2 c s \tanh [s(-c t+x)]) \text {, }
$$

$$
\begin{aligned}
& z_{3,1}(x, y, t) \\
& =\frac{-1}{\sigma^{2}}\left(\hbar y^{2} \operatorname{sech}^{2}[s(-c t+x)]\right. \\
& \times\left(k_{1} r^{2} \lambda \sin \left[2\left(k_{3} t+k_{1} x\right)\right]\right. \\
& \left.+s\left(-f s+2 r^{2} \lambda \cos ^{2}\left[k_{3} t+k_{1} x\right]\right) \tanh [s(-c t+x)]\right) \text {. }
\end{aligned}
$$

Obviously, for $\hbar=-1$, we obtained the same solutions as the one by the homotopy perturbation method in (20)-(22); we continue to evaluate six terms of (47) when $m=2,3$.

Using a Taylor series, then the closed form solutions yield as follows [23]:

$$
\begin{gathered}
q(x, y, t)=r \operatorname{sech}[s(x+y-c t)] \exp \left[i\left(k_{1} x+k_{2} y+k_{3} t\right)\right] \\
\phi(x, y, t)=f \tanh [s(x+y-c t)]
\end{gathered}
$$

where

$$
\begin{gathered}
c=k_{2}+\sigma^{2} k_{1}, \quad r=\sqrt{-\frac{2 k_{3}+\sigma^{2} k_{1}^{2}+k_{2}^{2}}{\lambda},} \\
s=\sqrt{\frac{2 k_{3}+\sigma^{2} k_{1}^{2}+k_{2}^{2}}{\lambda}}, \quad f=\frac{2 \sigma \sqrt{-\lambda}}{1-\sigma^{2}},
\end{gathered}
$$

$k_{1}, k_{2}$, and $k_{3}$ are arbitrary constants.
TABLE 4: The HAM results for $\phi(x, y, t)$ in comparison with the analytical solution with initial conditions (3).

\begin{tabular}{lc}
\hline$t$ & $|\phi e-\phi|$ \\
\hline-0.6 & 0 \\
-0.4 & 0 \\
-0.2 & $4 \times 10^{-10}$ \\
0 & 0 \\
0.2 & 0 \\
0.4 & $2 \times 10^{-10}$ \\
0.6 & 0 \\
\hline
\end{tabular}

TABLE 5: The HAM results for $q(x, y, t)$ in comparison with the analytical solution with initial conditions (5).

\begin{tabular}{lc}
\hline$t$ & $|q e-q|$ \\
\hline-0.6 & $9.861809932 \times 10^{-18}$ \\
-0.4 & $1.073356691 \times 10^{-17}$ \\
-0.2 & $1.161063556 \times 10^{-17}$ \\
0 & $1.248898384 \times 10^{-17}$ \\
0.2 & $1.336466963 \times 10^{-17}$ \\
0.4 & $1.423385326 \times 10^{-17}$ \\
0.6 & $1.509280934 \times 10^{-17}$ \\
\hline
\end{tabular}

TABLE 6: The HAM results for $\phi(x, y, t)$ in comparison with the analytical solution with initial conditions (5).

\begin{tabular}{lc}
\hline$t$ & $|\phi e-\phi|$ \\
\hline-0.6 & 0 \\
-0.4 & 0 \\
-0.2 & 0 \\
0 & 0 \\
0.2 & 0 \\
0.4 & 0 \\
0.6 & $5 \times 10^{-10}$ \\
\hline
\end{tabular}

\section{Comparing the HPM Results and the HAM Results with the Exact Solutions}

To demonstrate the convergence of the HPM, the results of the numerical example are presented and only few terms are required to obtain accurate solutions. Tables 1 and 2 show the absolute errors between the analytical solutions and the HPM solutions of the DS for the first three approximations with initial conditions (5) for $q(x, y, t), \phi(x, y, t)$ are very small with the present choice of $t$ at $x=50$ and $y=0.01$, when $k_{1}=0.1, k_{2}=0.03, k_{3}=-0.3, \sigma=I, \lambda=1$, and $m=0.1$. Tables $3,4,5$ and 6 help us to compare the HAM results for the first three approximations when $\hbar=-1$ with the analytical solution through the absolute errors. Both the analytical solutions, the HPM result, and the HAM result for $q(x, y, t)$ and $\phi(x, y, t)$ are plotted in Figures $1,2,3$, and 4 . 


\section{References}

[1] J. H. He, "Homotopy perturbation technique," Computer Methods in Applied Mechanics and Engineering, vol. 178, no. 3-4, pp. 257-262, 1999.

[2] H. A. Zedan, "Symmetry analysis of an integrable Ito coupled system," Computers and Mathematics with Applications, vol. 60, no. 12, pp. 3088-3097, 2010.

[3] J. H. He, "Asymptotology by homotopy perturbation method," Applied Mathematics and Computation, vol. 156, no. 3, pp. 591596, 2004.

[4] J. H. He, "The homotopy perturbation method for nonlinear oscillators with discontinuities," Applied Mathematics and Computation, vol. 151, no. 1, pp. 287-292, 2004.

[5] J. H. He, "Application of homotopy perturbation method to nonlinear wave equations," Chaos, Solitons and Fractals, vol. 26, no. 3, pp. 695-700, 2005.

[6] J. H. He, "Comparison of homotopy perturbation method and homotopy analysis method," Applied Mathematics and Computation, vol. 156, no. 2, pp. 527-539, 2004.

[7] M. Rafei and D. D. Ganji, "Explicit solutions of Helmholtz equation and fifth-order KdV equation using homotopy perturbation method," International Journal of Nonlinear Sciences and Numerical Simulation, vol. 7, no. 3, pp. 321-328, 2006.

[8] H. A. Zedan, "Exact solutions for the generalized KdV equation by using Backlund transformations," Journal of the Franklin Institute, vol. 348, no. 8, pp. 1751-1768, 2011.

[9] S. Abbasbandy, "Homotopy perturbation method for quadratic Riccati differential equation and comparison with Adomian's decomposition method," Applied Mathematics and Computation, vol. 172, no. 1, pp. 485-490, 2006.

[10] S. Abbasbandy, "Numerical solutions of the integral equations: homotopy perturbation method and Adomian's decomposition method," Applied Mathematics and Computation, vol. 173, no. 1, pp. 493-500, 2006.

[11] J. H. He, "Homotopy perturbation method for bifurcation of nonlinear problems," International Journal of Nonlinear Sciences and Numerical Simulation, vol. 6, no. 2, pp. 207-208, 2005.

[12] S. J. Liao, The proposed homotopy analysis technique fot the solution of nonlinear problems [Ph.D. thesis], Shanghai Jiao Tong University, 1992.

[13] S. Liao, Beyond Perturbation: Introduction to the Homotopy Analysis Method, CRC Series: Modern Mechanics and Mathematics, Chapman \& Hall/CRC, Boca Raton, Fla, USA, 2003.

[14] S. Liao, "On the homotopy analysis method for nonlinear problems," Applied Mathematics and Computation, vol. 147, no. 2, pp. 499-513, 2004.

[15] S. J. Liao, "Comparison between the homotopy analysis method and homotopy perturbation method," Applied Mathematics and Computation, vol. 169, no. 2, pp. 1186-1194, 2005.

[16] S. Abbasbandy, "The application of homotopy analysis method to solve a generalized Hirota-Satsuma coupled KdV equation," Physics Letters A, vol. 361, no. 6, pp. 478-483, 2007.

[17] S. Abbasbandy, "The application of homotopy analysis method to nonlinear equations arising in heat transfer," Physics Letters A, vol. 360, no. 1, pp. 109-113, 2006.

[18] H. A. Zedan, " $\left(G^{\prime} / G\right)$-expansion method for the generalized Zakharov equations," Ricerche di Matematica, vol. 60, no. 2, pp. 203-217, 2011.

[19] M. Sajid and T. Hayat, "Comparison of HAM and HPM methods in nonlinear heat conduction and convection equations,"
Nonlinear Analysis: Real World Applications, vol. 9, no. 5, pp. 2296-2301, 2008.

[20] M. M. Rashidi, G. Domairry, and S. Dinarvand, "Approximate solutions for the Burger and regularized long wave equations by means of the homotopy analysis method," Communications in Nonlinear Science and Numerical Simulation, vol. 14, no. 3, pp. 708-717, 2009.

[21] H. A. Zedan and S. M. Al-Tuwairqi, "Painlevé analysis of generalized zakharov equations," Pacific Journal of Mathematics, vol. 247, no. 2, pp. 497-510, 2010.

[22] A. Davey and K. Stewartson, "On three-dimensional packets of surface waves," Proceedings of the Royal Society London A, vol. 338, pp. 101-110, 1974.

[23] H. A. Zedan and S. S. Tantawy, "Solution of Davey-stewartson equations by homotopy perturbation method," Computational Mathematics and Mathematical Physics, vol. 49, no. 8, pp. 1382$1388,2009$.

[24] S. J. Liao, "An approximate solution technique not depending on small parameters: a special example," International Journal of Non-Linear Mechanics, vol. 30, no. 3, pp. 371-380, 1995.

[25] S. J. Liao, "A kind of approximate solution technique which does not depend upon small parameters-II: an application in fluid mechanics," International Journal of Non-Linear Mechanics, vol. 32, no. 5, pp. 815-822, 1997.

[26] S. J. Liao, "An explicit, totally analytic approximate solution for Blasius' viscous flow problems," International Journal of NonLinear Mechanics, vol. 34, no. 4, pp. 759-778, 1999. 


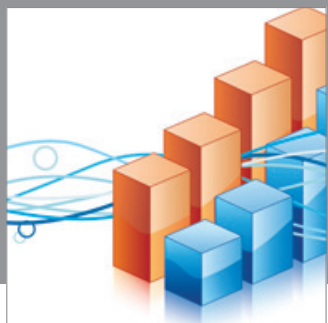

Advances in

Operations Research

mansans

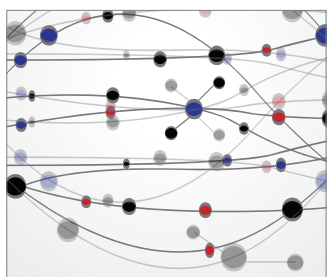

The Scientific World Journal
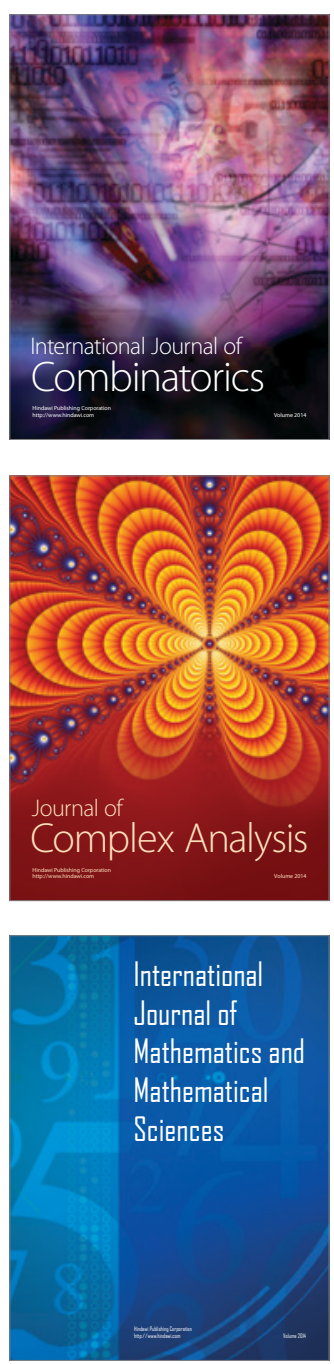
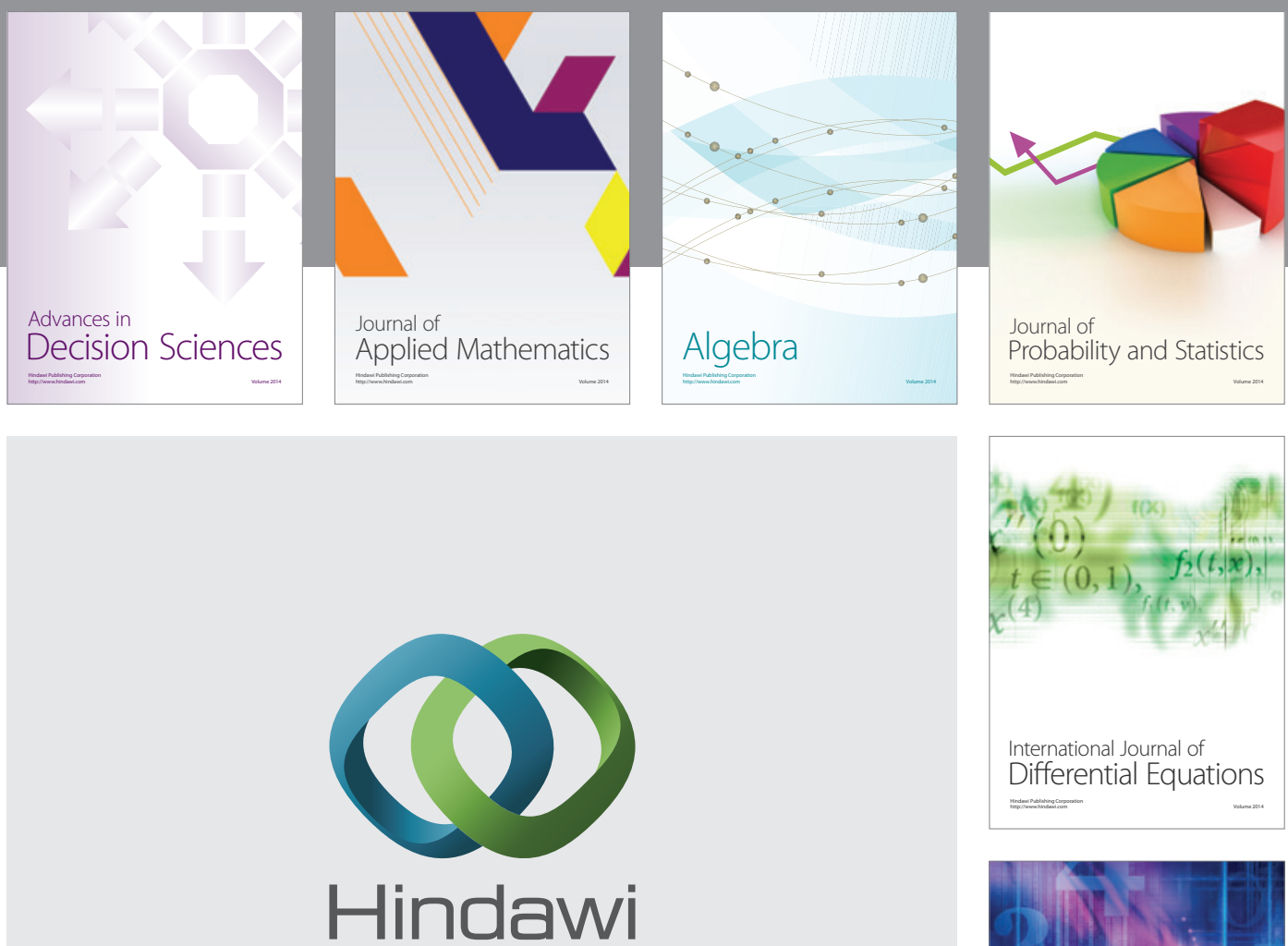

Submit your manuscripts at http://www.hindawi.com
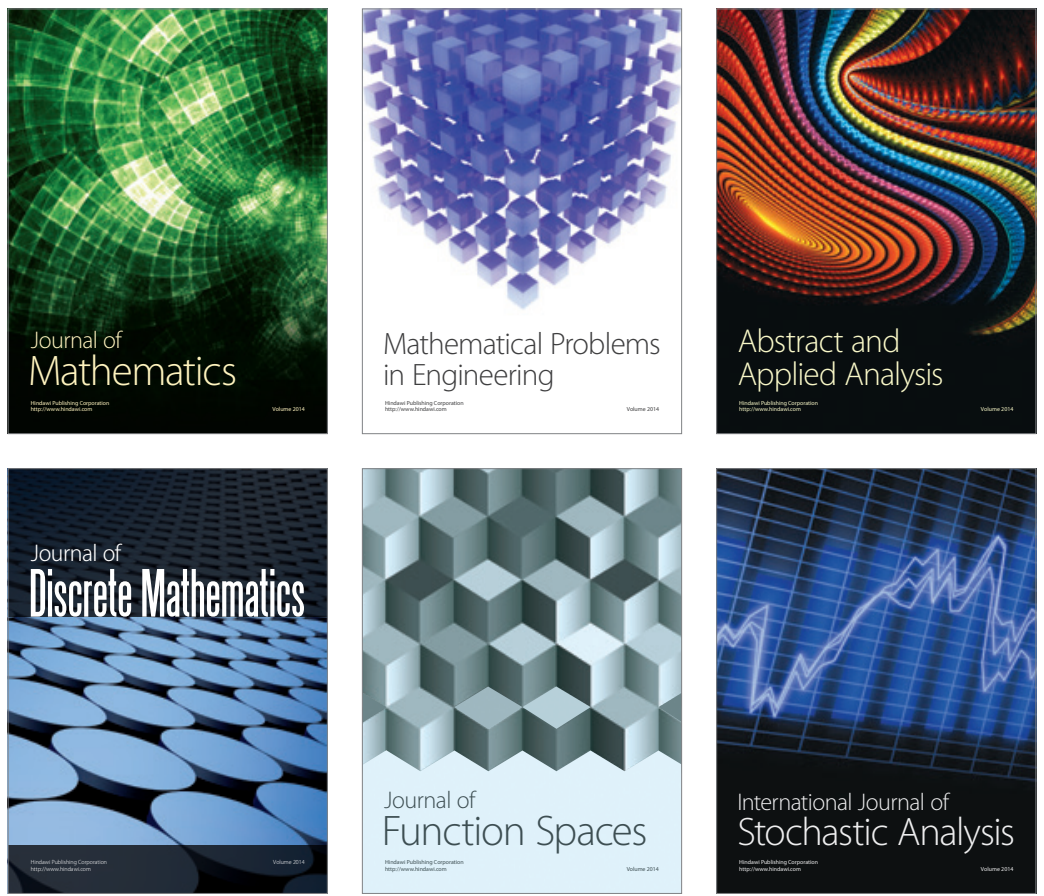

Journal of

Function Spaces

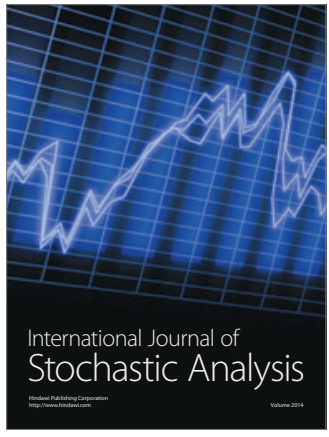

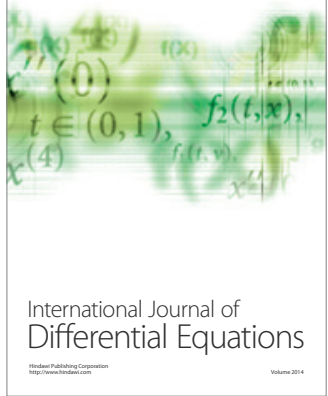
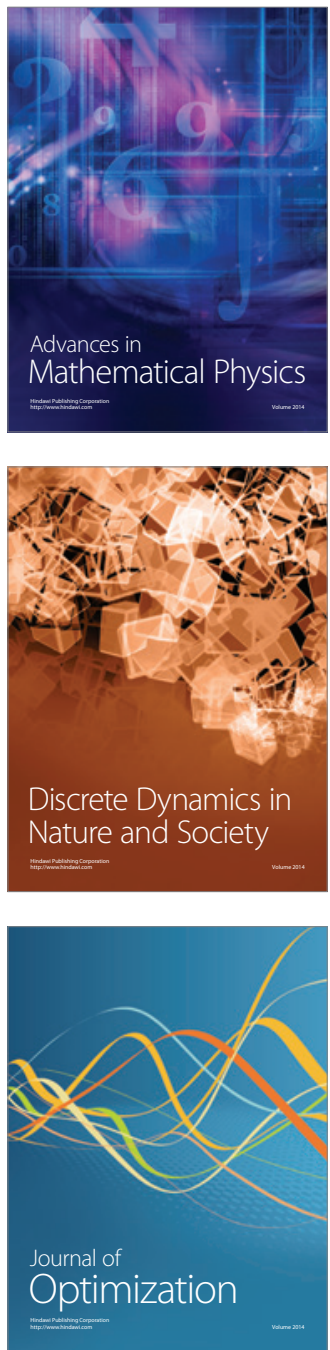